Education Development Programme was developed and delivered to specialist nurses. We report on the evaluation of the programme.

Methods A mixed methods evaluation was adopted, with purposive sampling of 20 participants who completed the Education Development Programme and agreed to participate in the evaluation. Each participant provided a pre- and post- test score on a Likert scale for willingness and confidence to teach junior staff, staff at the same grade and medical staff.

Results An improvement was found in all participants' reported willingness and confidence to teach all grades of staff, particularly medical staff. From the focus groups two main themes were identified; learning to teach and building skills to change practice. Participants reported greater confidence and preparedness for their teaching roles. Their growth in confidence and the practical skills they have gained have equipped the participants to be able to teach a diverse workforce.

Conclusions It cannot be assumed that specialist staff, with teaching in their role, have the skills to facilitate learning. This programme offers a potential method of improving facilitation skills. Specialist staff with teaching responsibilities should be provided with education and training to develop their teaching and facilitation skills.

Funding Cheshire \& Merseyside Palliative and End of Life Network Education Strategy Group.

\section{P-57 USING SIMULATION FOR DNACPR DISCUSSION TRAINING: A NOVEL ROLE FOR HOSPICE VOLUNTEERS IN EDUCATION}

${ }^{1}$ Mursheda Chowdhury, ${ }^{1}$ Niamh Hanna, ${ }^{2}$ Alastair Lowe, 'Jane Marquesen, ${ }^{1}$ Rosie Tucker, ${ }^{1}$ Jill Beckhelling. 'St Michael's Hospice, St Leonards on Sea, UK; ${ }^{2}$ East Sussex Healthcare NHS Trust, St Leonards on Sea, UK

\subsection{6/bmjspcare-2019-HUKNC.8}

Background Evidence shows patient dissatisfaction regarding healthcare professionals' discussions of DNACPR (Do Not Attempt Cardio-pulmonary Resuscitation) and Ceilings of Treatment. Often the reality is that healthcare professionals are having to broach these conversations in less than ideal circumstances. Increasingly, the responsibility falls on nurses and new trainees with no opportunity to get feedback on the impact on patients and to learn in a constructive way from experience. We used hospice volunteers to take part in training two different groups (a) Hospice Community Clinical Nurse Specialists (CNSs) and (b) Foundation Year (FY) Doctors. The volunteers took part as simulated patients/relatives and provided feedback.

Aims The aim was to increase the confidence levels of the FY Doctors and CNSs through conducting DNACPR and Ceilings of Treatment discussions in a safe environment. For CNSs, there was an additional aim - to evaluate competency using simulation after a simulated practice session.

Methods Bespoke scenarios were written for each of the two groups, tailored to the likely clinical circumstances in which they would be having these discussions. Volunteers were recruited from the existing pool of hospice volunteers. The volunteers were briefed and shown the Simulation Suite in advance. Confidence levels were completed by participants pre- and post each session. A competency framework was written against which to evaluate the CNSs' performance.

Results In both groups, $100 \%$ of the participants stated that their confidence levels increased. Both groups commented on the usefulness of feedback from the volunteers. $100 \%$ of the CNSs achieved the required competency after the practice session.

Conclusion Simulation is an effective way of improving the confidence levels of healthcare professionals in conducting DNACPR and Ceilings of Treatment discussions. Simulation can be used to conduct evaluation of competency. Hospice volunteers are a useful resource in educational initiatives using simulation and can provide dynamic feedback for healthcare professionals to aid reflective learning.

\section{P-58 'WE REALLY DO WANT TO BE INVOLVED-IT'S A YES FROM US' PATIENT INVOLVEMENT IN THE CLASSROOM}

Liz Watson, Stevan Stratford, Heather Calver. St Barnabas House, Worthing, UK

\subsection{6/bmjspcare-2019-HUKNC.82}

The St Barnabas House Education Programme is comprehensive and accessed by hospice staff and external health and social care professionals. We work in partnership with patients and relatives on different study days to provide a focus on what really matters to them. They are invited to be involved by the clinicians in practice and they meet a member of the Education Team. A discussion takes place about how they would like to be involved, this has included the production of short films, discussions, storytelling, art work and group work in the classroom. The stories are very real in their sincerity and sensitivity highlighting many different key issues, particularly the importance of empathetic and honest communication and the very real value of humanity.

Working in partnership we support the patient/relative to feel as empowered and comfortable as possible. This is followed through as part of the working agreement with delegates at the start of the study day and ensures safety for everyone.

Films have included 'The Reality of Living with Motor Neurone Disease' and how addressing the little things make such a difference to the patient. A lady, diagnosed with advanced Parkinson's disease made a fabulous film demonstrating the importance of continuing to roller skate despite her severe physical disability. Another lady expressed her difficulty to communicate her feelings through the use of paintings and this was enhanced by art work created by her husband. The richness of the patient/relatives narrative is very powerful and has raised many pertinent issues for discussion in the classroom with the patient/relative.

The evaluations have been very encouraging, highlighting how the patient/relatives story will positively impact on professional clinical practice. A very welcomed and unexpected result has been how valuable the patients/relatives have reported on the experience for them, an opportunity to make meaningful change, influencing the future through evolution. 